\title{
Brownian pump in nonlinear diffusive media
}

\author{
Bao-quan $\mathrm{Ai}^{1 *}$ and Liang-gang $\mathrm{Liu}^{2}$ \\ ${ }^{1}$ Institute for Condensed Matter Physics, \\ School of Physics and Telecommunication Engineering, \\ South China Normal University, 510006 Guangzhou, China \\ 2 Faculty of Information Technology , \\ Macau University of Science and Technology, Macao
}

(Dated: November 15, 2018)

\begin{abstract}
A Brownian pump in nonlinear diffusive media is investigated in the presence of an unbiased external force. The pumping system is embedded in a finite region and bounded by two particle reservoirs. In the adiabatic limit, we obtain the analytical expressions of the current and the pumping capacity as a function of temperature for normal diffusion, subdiffusion and superdiffusion. It is found that important anomalies are detected in comparison to the normal diffusion case. The superdiffusive regime, compared with the normal one, exhibits an opposite current for low temperatures. In subdiffusive regime, the current may become forbidden for low temperatures and negative for high temperatures.
\end{abstract}

PACS numbers: 05.60.-k, 87.16.Uv, 05. 06. Cd, 02. 50. Ey

Keywords: Brownian pump, nonlinear diffusive media, concentration ratio.

*Email: aibq@hotmail.com 


\section{INTRODUCTION}

Pumping is an active nonequilibrium transport process in which fluctuations play a very important role. Some pumps have already been investigated in the literature[1 [6]. Kosztin and Schulten [1] studied the fluctuation-driven molecular transport through an asymmetric potential pump and three transport mechanisms: driven by potential gradient, by an external periodic force and by nonequilibrium fluctuations [2]. Moskalets and Buttiker [3] studied heat fluxes in adiabatic quantum pumps and developed a approach to the kinetics for an arbitrary relation of pump frequency and temperature. Arrachea and coworkers[4] investigated the heat transport and the power developed the local driving fields on an quantum system coupled macroscopic reservoirs. They identified two generic interesting mechanisms: directed heat transport between reservoirs induced by the ac potentials and at slow driving, two oscillating out of phase forces perform work against each other. Nonadiabatic electron heat pump was investigated by Rey and coworkers [5]. They presented a mechanism for extracting heat metallic conductors based on the energy-selective transmission of electrons through a spatially asymmetric resonant structure subject to ac driving.

Recently, Sancho and Gomez-marin [6] presented a model for a Brownian pump powered by a flashing ratchet mechanism. The pumping device was embedded in a finite region and bounded by particle reservoirs. Their emphasis is on finding what concentration gradient the pump can maintain. All of the above examples have been formulated within a standard Brownian framework, for which diffusion properties are normal, that is, with the mean quadratic displacement growing linearly with time $t$. However, it is well know that there are media signaled by a $t^{\mu}$ growth of the squared dispersion with $\mu \neq 1$. An important class is given by the " porous medium " equation [7-10], which, in the one-dimensional problem and in the absence of external forces, can cast in the form

$$
\partial_{t} \rho=D \mu \partial_{x}\left(\rho^{\mu-1} \partial_{x} \rho\right)
$$

where $\rho$ is the density of the diffusing substance, $x$ is a dimensionless coordinate representing a bond length, angle, or any other chemical or physical state variable, $t$ is the dimensionless time, and $D$ is diffusive constant. The mean quadratic deviation follows the law $\left\langle x(t)^{2}\right\rangle \propto$

$t^{\frac{2}{1+\mu}}$. The transport is subdiffusive for $\mu>1$, normal for $\mu=1$ and superdiffusive for $0<\mu<1$. Many physical systems are well-described by this class of processes: percolation 
of gases in porous media [11], dispersion of biological populations [12], grain segregation [13], fluxes in plasma [14] and nonextensive statistics [15].

The present work is extend to the study of the Brownian pump to the case of the anomalous diffusion. We emphasize on finding how particles can be pumped through a cell membrane from a particle reservoir at low concentration to one at the same or higher concentration in a nonlinear diffusive media.

\section{GENERAL ANALYSIS}

We consider a Brownian pump which is located in a finite region and transports particles across the barrier against the concentration gradients by using an unbiased external force. Here, we model the device as a pumping system in which overdamped Brownian particles moving in a asymmetric finite potential in the presence of an unbiased external force. The potential is embedded in a finite region $[0, L]$,

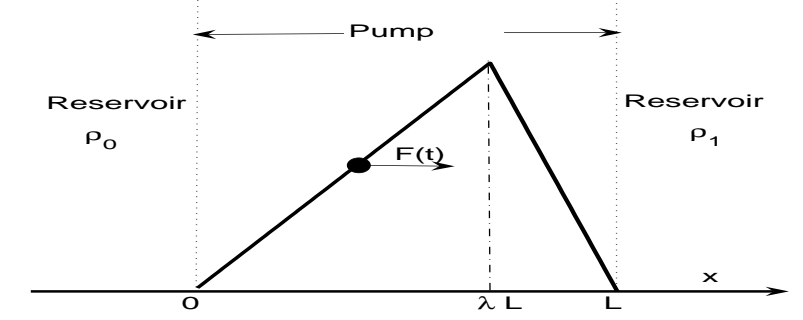

FIG. 1: Scheme of a Brownian pump: A spatially asymmetric potential $U_{0}(x)($ defined in Eq. (2)) is embedded in a finite region and bounded by two particle reservoirs of concentrations $\rho_{0}$ and $\rho_{1}$. $L$ and $\lambda$ is the length and the asymmetric parameter of the potential, respectively. The particles are driven by an unbiased external force $F(t)$, defined by Eq. (3).

$$
U_{0}(x)= \begin{cases}Q \frac{x}{\lambda L}, & 0 \leq x<\lambda L \\ Q \frac{L-x}{(1-\lambda) L}, & \lambda L \leq x \leq L,\end{cases}
$$

where $Q$ is amplitude of the potential and $\lambda$ is its asymmetric parameter. $F(t)$ is an unbiased external force and satisfies

$$
F(t)=\left\{\begin{array}{l}
F_{0}, \quad n \tau \leq t<n \tau+\frac{1}{2} \tau \\
-F_{0}, \quad n \tau+\frac{1}{2} \tau<t \leq(n+1) \tau,
\end{array}\right.
$$


where $\tau$ is the period of the unbiased force and $F_{0}$ is its magnitude.

The correlated anomalous diffusion can be described through the following nonlinear Fokker-Planck equation [8-10]:

$$
\begin{gathered}
\frac{\partial}{\partial t} \rho(x, t)=\frac{\partial}{\partial x}\left[U^{\prime}(x, t) \rho(x, t)\right]+D \frac{\partial^{2}}{\partial x^{2}} \rho^{\mu}(x, t)=-\frac{\partial j(x, t)}{\partial x}, \\
U(x, t)=U_{0}(x)-F(t) x,
\end{gathered}
$$

where $(\mathrm{x}, \mathrm{t})$ is a dimensionless $1+1$ space time and $D=\frac{k_{B} T}{\eta} \cdot k_{B}$ is the Boltzmann constant, $T$ is the temperature and $\eta$ is friction coefficient. The prime stands for the derivative with respect to the space variable $x . j(x, t)$ is the probability current and $\rho(x, t)$ is the particle concentration. This nonlinear equation yields anomalous diffusion when $\mu \neq 1$ : subdiffusion for $\mu>1$ and superdiffusion for $0<\mu<1$.

\section{A. Normal diffusion}

For the normal diffusive case $\mu=1$, Eq. (4) recovers the ordinary Fokker-Planck equation and $j(x, t)$ satisfies

$$
j(x, t)=-U^{\prime}(x, t) \rho(x, t)-D \frac{\partial}{\partial x} \rho(x, t) .
$$

The density $\rho(x, t)$ follows a first order non homogenous linear differential equation, whose formal solution is

$$
\rho(x, t)=\exp \left[-\int_{0}^{x} \frac{U^{\prime}(z, t)}{D} d z\right]\left\{c_{0}-\frac{j}{D} \int_{0}^{x} d z \exp \left[\int_{0}^{z} \frac{U^{\prime}(y, t)}{D} d y\right]\right\} .
$$

If $F(t)$ changes very slowly with respect to $t$, namely, its period is longer than any other time scale of the system, there exists a quasistatic state. In the steady state, the concentration is just a function of space thus the flux becomes a constant $j$. Though, unlike typical Brownian motors [6, 16, 17], the boundary conditions are not periodic nor the normalized condition is imposed, the unknown constant $c_{0}$ and $j$ can be found by imposing the left reservoir concentration $\rho_{0} \equiv \rho(0)$ and the right concentration $\rho_{1} \equiv \rho(L)$ as fixed boundary conditions. We can find that $c_{0}=\rho_{0}$ and

$$
j\left(F_{0}\right)=\frac{D\left\{\rho_{0}-\rho_{1} \exp \left[-\frac{F_{0} L}{D}\right]\right\}}{\int_{0}^{L} \exp \left[\frac{U_{0}(x)-F_{0} x}{D}\right] d x}=\frac{D}{I\left(F_{0}\right)}\left\{\rho_{0}-\rho_{1} \exp \left[-\frac{F_{0} L}{D}\right]\right\},
$$


where

$$
I\left(F_{0}\right)=\frac{\lambda L D}{Q-F_{0} \lambda L}\left[\exp \left(\frac{Q-F_{0} \lambda L}{D}\right)-1\right]+\frac{D(\lambda-1) L}{Q-F_{0}(\lambda-1) L}\left[\exp \left(-\frac{F_{0} L}{D}\right)-\exp \left(\frac{Q-F_{0} \lambda L}{D}\right)\right] .
$$

The average current is

$$
J=\frac{1}{\tau} \int_{0}^{\tau} j(F(t)) d t=\frac{1}{2}\left[j\left(F_{0}\right)+j\left(-F_{0}\right)\right] .
$$

For studying the pumping capacity, we consider the situation in which $J$ tends to zero which corresponds the case in which the pump in maintaining the maximum concentration difference between the two reservoirs across the barrier with no net leaking of particle. This situation is analogous the stalling force in Brownian motors. From Eqs. (8-10), we can obtain

$$
\frac{\rho_{1}}{\rho_{0}}=\frac{I\left(F_{0}\right)+I\left(-F_{0}\right)}{e^{\frac{F_{0} L}{D}} I\left(F_{0}\right)+e^{-\frac{F_{0} L}{D}} I\left(-F_{0}\right)} .
$$

\section{B. Anomalous diffusion}

When $\mu$ in Eq. (4) is not equal to 1, we have

$$
j(x, t)=-U^{\prime}(x, t) \rho(x, t)-D \frac{\partial}{\partial x} \rho^{\mu}(x, t) .
$$

In this case, the explicit expression for $\rho(x, t)$ cannot be extracted from above equation by using the same method as the case of $\mu=1$. Here, we use the method presented by Zhao and coworkers [10] to derive the expressions for $j$ and $\rho$. Let us assume

$$
\rho_{m}(x, t)=G(x, t) \rho^{\mu}(x, t), \quad j_{m}(x, t)=G(x, t) j(x, t),
$$

where $G(x, t)$ is a factor function. We assume that there exists the proper $\rho_{m}(x, t)$ and $j_{m}(x, t)$ satisfying diffusion law,

$$
j_{m}(x, t)=-D \frac{\partial}{\partial x} \rho_{m}(x, t)
$$

From Eqs. (13) and (14), we have

$$
j(x, t)=-D \rho^{\mu}(x, t) \frac{\partial}{\partial x}[\ln G(x, t)]-D \frac{\partial}{\partial x} \rho^{\mu}(x, t)
$$

Comparing Eq. (12) with Eq. (15), we have

$$
G(x, t)=\exp \left[\frac{\phi_{\mu}(x, t)}{D}\right]
$$


where

$$
\phi_{\mu}(x, t)=\int U^{\prime}(x, t) \rho^{\mu-1}(x, t) d x .
$$

It is obvious that $\phi_{\mu}$ represents an effective $\rho$-dependent potential resulting from the nonlinear diffusive media. For a quasistatic state, we can get the static solution of Eq. (4)

$$
\rho_{s}(x)=[\Psi(x)]_{+}^{\frac{1}{\mu-1}}
$$

where $[f]_{+}=\max \{f, 0\}$ and

$$
\Psi(x)=\rho_{0}^{\mu-1}-\frac{\mu-1}{D \mu} U(x) .
$$

From Eqs. (17-19), we can obtain

$$
\phi_{\mu}(x, t)=\frac{\mu D}{1-\mu} \ln [\Psi(x)] .
$$

So we can rewrite the Eq. (15) as

$$
j(x, t)=-D \exp \left[-\frac{\phi_{\mu}(x, t)}{D}\right] \frac{\partial}{\partial x}\left[\exp \left(\frac{\phi_{\mu}(x, t)}{D}\right) \rho^{\mu}(x, t)\right] .
$$

Integrating over $x$ from 0 to $x$, we have

$$
\frac{j}{D} \int_{0}^{x} \exp \left[\frac{\phi_{\mu}(x, t)}{D}\right] d x=-\int_{0}^{x} \frac{\partial}{\partial y}\left[\exp \left(\frac{\phi_{\mu}(y, t)}{D}\right) \rho^{\mu}(y, t)\right] d y .
$$

So we can get the expression for $\rho^{\mu}(x, t)$

$$
\rho^{\mu}(x, t)=\frac{\exp \left[\frac{\phi_{\mu}(0, t)}{D}\right] \rho^{\mu}(0, t)-\frac{j}{D} \int_{0}^{x} \exp \left[\frac{\phi_{\mu}(x, t)}{D}\right] d x}{\exp \left[\frac{\phi_{\mu}(x, t)}{D}\right]} .
$$

The constant $j$ can be found by imposing the left reservoir concentration $\rho_{0} \equiv \rho(0)$ and the right concentration $\rho_{1} \equiv \rho(L)$ as fixed boundary conditions,

$$
j\left(F_{0}\right)=\frac{\theta\left(F_{0}\right) D}{I\left(F_{0}\right)}\left[\rho_{0}^{\mu}-M\left(F_{0}\right) \rho_{1}^{\mu}\right]
$$

where

$$
M\left(F_{0}\right)=\left[\rho_{0}^{\mu-1}+\frac{\mu-1}{D \mu} F_{0} L\right]^{\frac{\mu}{1-\mu}}
$$

and

$$
\begin{aligned}
I\left(F_{0}\right) & =\int_{0}^{L}[\Psi(x)]^{\frac{\mu}{1-\mu}} d x \\
& =\frac{\mu-1}{A}\left[\left(\rho_{0}^{\mu-1}-A \lambda L\right)^{\frac{1}{1-\mu}}-\rho_{0}\right]+\frac{1-\mu}{B}\left[(C+B L)^{\frac{1}{1-\mu}}-(C+B \lambda L)^{\frac{1}{1-\mu}}\right],
\end{aligned}
$$


where $A=\frac{(\mu-1)\left(Q-F_{0} \lambda L\right)}{D \mu \lambda}, B=\frac{(\mu-1)\left[Q+F_{0}(1-\lambda) L\right]}{D \mu(1-\lambda) L}$ and $C=\rho_{0}^{\mu-1}-\frac{(\mu-1) Q}{D \mu(1-\lambda)}$.

It must be pointed out that when $\Psi(x)$ is not always positive there exists a cut off of probability. A cutoff condition (Tsallis cutoff) yields regions with null probability. This is because a cutoff of the stationary solution Eq. (18) restricts the attainable space. Since the probability of particles visiting the special regions is null, the particles can not pass across pump, then there is no current. In order to describe this cut off a function $\theta\left(F_{0}\right)$ is defined by

$$
\theta\left(F_{0}\right)=\left\{\begin{array}{l}
1, \Psi(x)>0, \text { for all values of } x \\
0, \text { otherwise }
\end{array}\right.
$$

Similarly, the average current is

$$
J=\frac{1}{2}\left[j\left(F_{0}\right)+j\left(-F_{0}\right)\right]
$$

From Eqs. (24-28), we can obtain the maximum concentration ratio for $J=0$ and $\rho_{0}=1$,

$$
\frac{\rho_{1}^{\mu}}{\rho_{0}^{\mu}}=\frac{\theta\left(F_{0}\right) I\left(-F_{0}\right)+\theta\left(-F_{0}\right) I\left(F_{0}\right)}{\theta\left(F_{0}\right) I\left(-F_{0}\right) M\left(F_{0}\right)+\theta\left(-F_{0}\right) I\left(F_{0}\right) M\left(-F_{0}\right)} .
$$

\section{RESULTS AND DISCUSSIONS}

Our study focus on the current and the maximum concentration ratio at $J=0$ for normal diffusion, subdiffusion and superdiffusion. For simplicity, we take $k_{B}=1, \eta=1$ and $L=1$ throughout the study.

\section{A. Normal diffusion $\mu=1$}

In Fig. 2, we present current as a function of temperature $T$ for normal diffusion $(\mu=1.0)$. For lower values of $\rho_{1}$ the current is larger. When $\rho_{1}$ is more than $\rho_{0}$, the current is negative for high temperatures. When $T \rightarrow 0$, the particles cannot pass over the potential barrier and the current tends to zero. When $T \rightarrow \infty$, the ratchet effect disappears, the transport is dominated by the concentration difference and the particles move to the left. Therefore, there exists an optimized value of $T$ at which the current takes its positive maximum value. It is note that the similar behavior is also presented in Ref. 6 . 


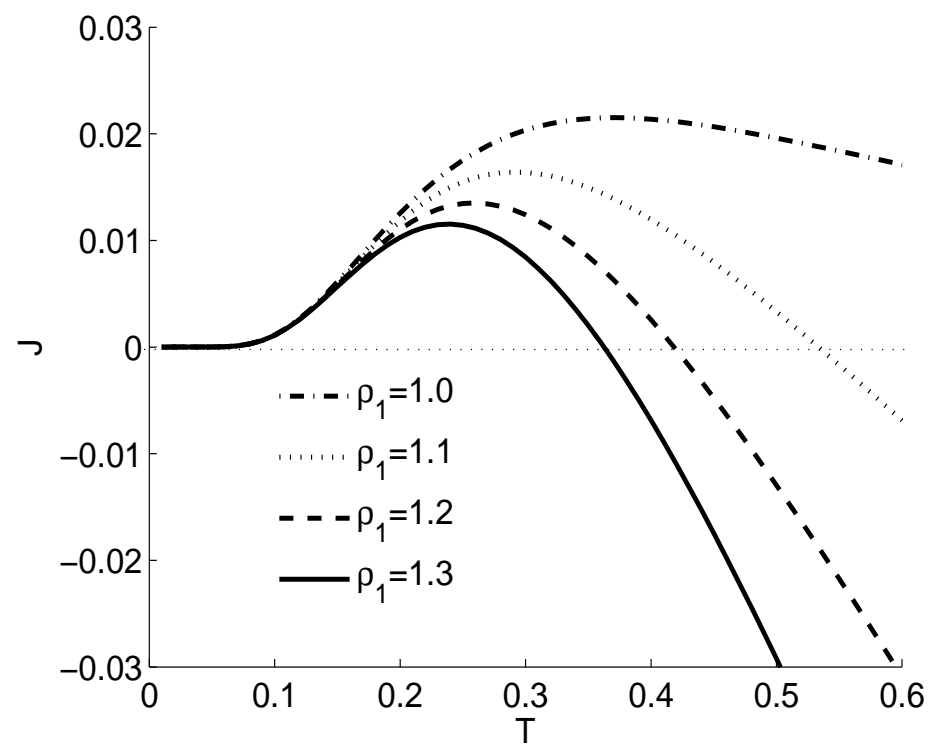

FIG. 2: Current $J$ versus temperature $T$ for different values of $\rho_{1}$ at $Q=1, \lambda=0.9, F_{0}=0.5$, $\rho_{0}=1.0$, and $\mu=1$.

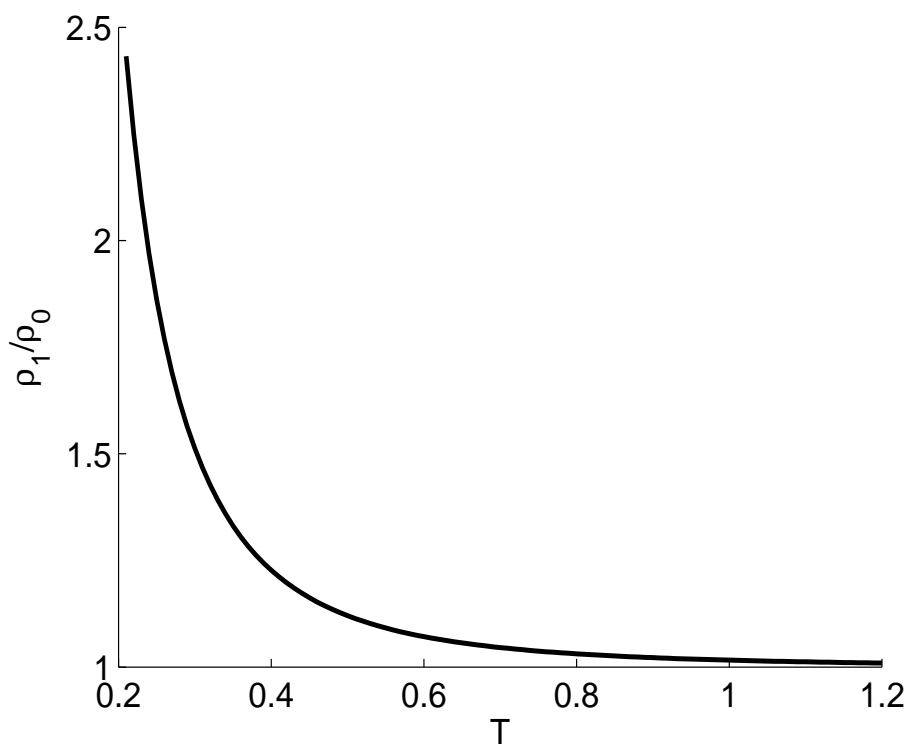

FIG. 3: Concentration ratio $\rho_{1} / \rho_{0}$ as a function of temperature $T$ for $J=0$ at $Q=1, \lambda=0.9$, $F_{0}=0.5$, and $\mu=1$. 
Figure 3 shows the ratio $\rho_{1} / \rho_{0}$ as a function of temperature $T$ for normal diffusion $(\mu=1)$. When $T \rightarrow 0$, no particle can pass over the barrier, thus $\rho_{1} / \rho_{0} \rightarrow \infty$. As temperature $T$ is increased, the ratchet effect reduces and the pumping capacity decreases. Surprisingly, the temperature corresponding to the maximum current is not the same the temperature at which the concentration ratio for zero current is maximum. This cause for this is that zero current induces the maximum concentration ratio, not the minimum one.

\section{B. Subdiffusion $\mu>1$}

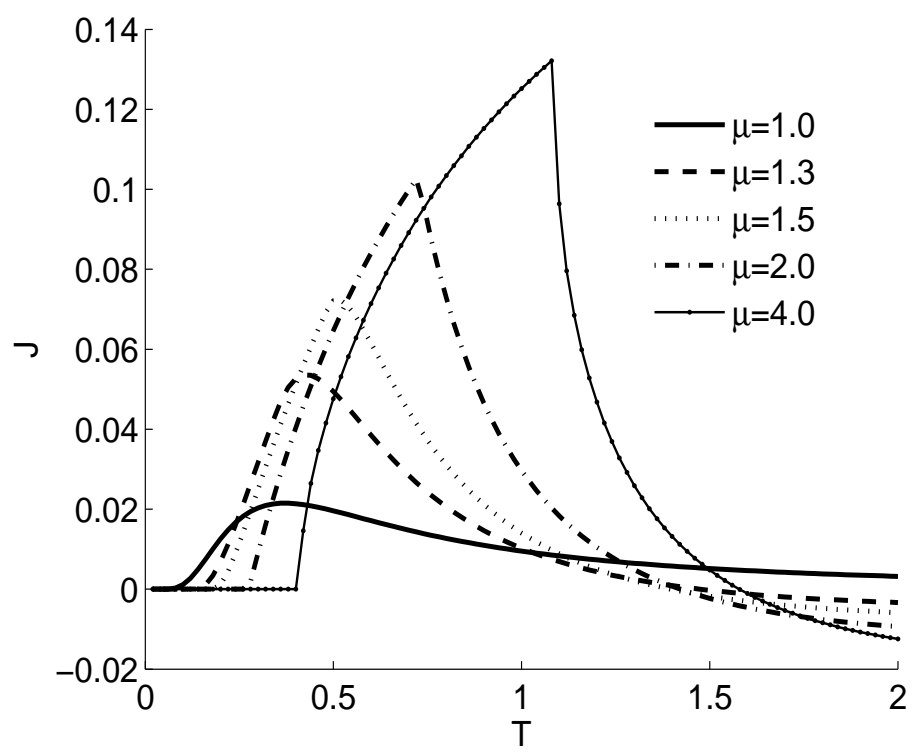

FIG. 4: Current $J$ as a function of temperature $T$ for different values of $\mu$ at $Q=1, \lambda=0.9$, $F_{0}=0.5, \rho_{0}=1.0$, and $\rho_{1}=1.0$.

Figure 4 shows the current $J$ versus temperature $T$ for subdiffusion $(\mu>1)$ at $\rho_{1}=$ $\rho_{0}=1.0$. The curve for normal diffusion is observed to be bell shaped. However, as $\mu$ is increased, the curve becomes to be not smooth. There exist two values of temperature at which the curve has inflexions: the lowest temperature to obtain a positive current, the optimized temperature to obtain a maximum current. For low temperatures, there lies a finite temperature only above which the particle can pass over the barrier, otherwise the particles will be confined in both reservoirs. For high temperatures, the current is negative. However, when $T \rightarrow \infty$, the current will approach to zero. 


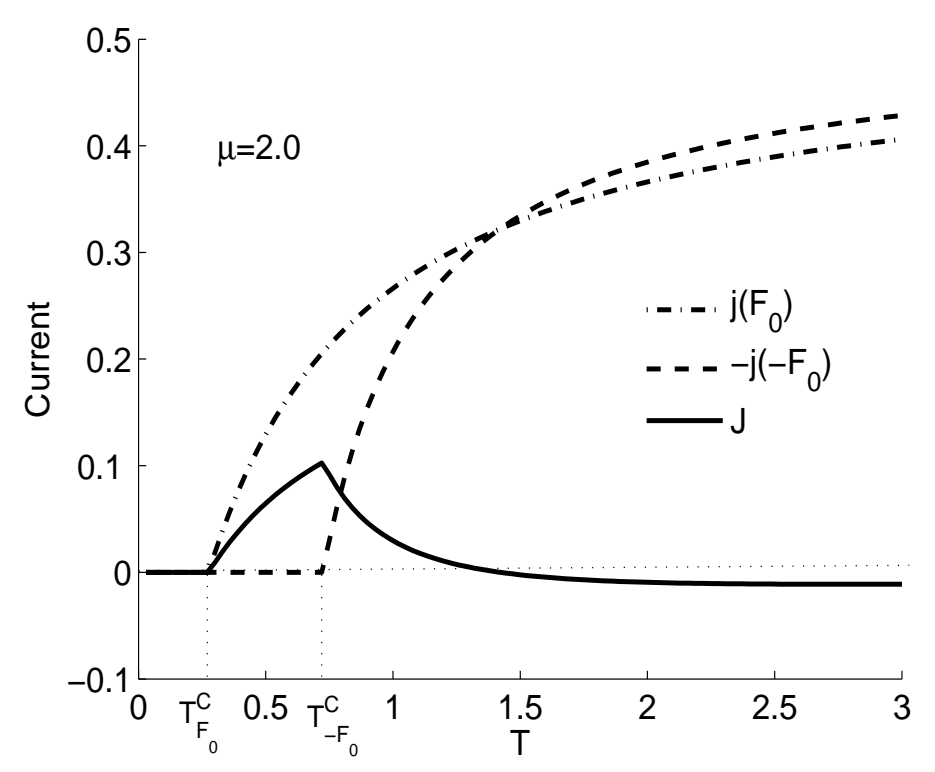

FIG. 5: Currents $j\left(F_{0}\right),-j\left(-F_{0}\right)$ and $J$ versus temperature $T$ for $\mu=2.0$ at $Q=1, \lambda=0.9$, $F_{0}=0.5, \rho_{0}=1.0$, and $\rho_{1}=1.0$. The temperatures for the two inflexions are $T_{F_{0}}^{C}=0.225$, $T_{-F_{0}}^{C}=0.725$.

In order to illustrate the transport behavior for subdiffusion, the currents $j\left(F_{0}\right),-j\left(-F_{0}\right)$ and $J$ as a function of $T$ for $\mu=2.0$ are shown in Fig. 5. From Eq. (19) and (27), we can find that there exist two values of temperature

$$
T_{\mp F_{0}}^{C}=\frac{\mu-1}{\mu}\left(Q \pm F_{0} \lambda\right)
$$

only above which $\Psi(x)$ is always positive, otherwise $\Psi(x)$ may be negative. Therefore, when $T \leq T_{\mp F_{0}}^{C}$, the state space becomes disconnected and crossings become forbidden. The local current $j$ tends to zero. This is because a cut off of the stationary solution of Eq. (18) restricts the attainable space. From the figure, we can see that both $j\left(F_{0}\right)$ and $j\left(-F_{0}\right)$ are zero and $J=0$ for $T<T_{F_{0}}^{C} ; j\left(F_{0}\right)$ is positive, $j\left(-F_{0}\right)$ is zero and $J=\frac{1}{2} j\left(F_{0}\right.$ for $T_{F_{0}}^{C}<T<T_{-F_{0}}^{C} ; j\left(F_{0}\right)$ is positive, $j\left(-F_{0}\right)$ is negative and $J=\frac{1}{2}\left[j\left(F_{0}\right)+j\left(-F_{0}\right)\right]$ for $T>T_{-F_{0}}^{C}$. The first inflexion is at $T_{F_{0}}^{C}$ and the second one is at $T_{-F_{0}}^{C}$.

Figure 6 shows the concentration ratio $\rho_{1} / \rho_{0}$ as a function of $T$ for subdiffusion $(\mu=1.5$, 2.0, and 4.0). The ratio $\rho_{1} / \rho_{0}$ decreases with increasing $T$. The curve is not smooth and there exists a inflexion at $T=T_{F_{0}}^{C}$. For low temperatures, the ratio $\rho_{1} / \rho_{0}$ decreases with 


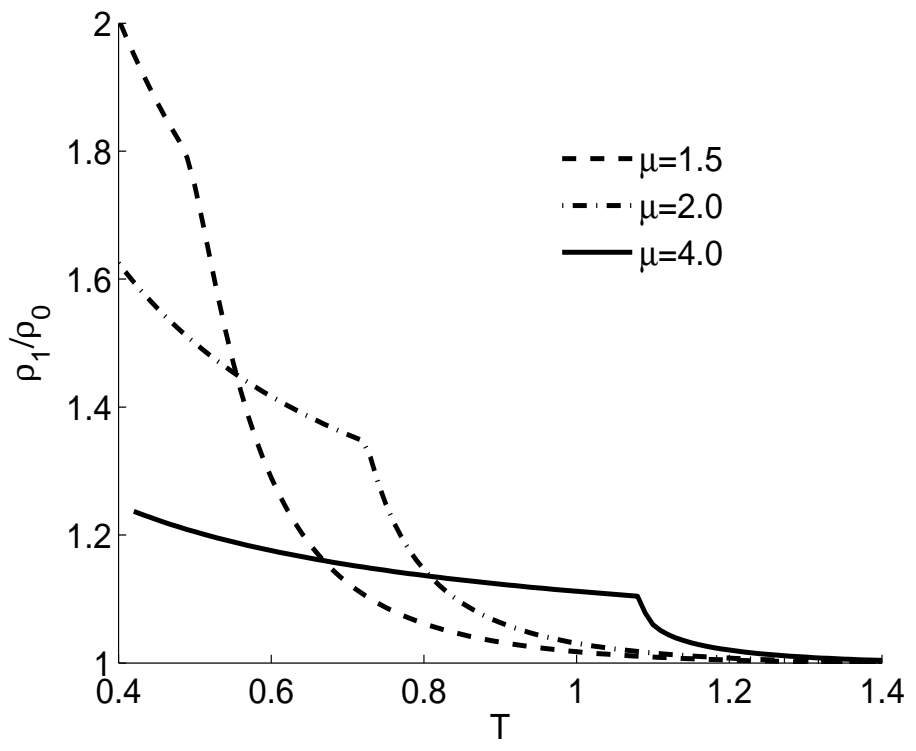

FIG. 6: Concentration ratio $\rho_{1} / \rho_{0}$ as a function of temperature $T$ for $J=0$ at $Q=1, \lambda=0.9$, $F_{0}=0.5, \rho_{0}=1.0$, and $\mu=1.5,2.0,4.0$.

increasing $\mu$, while it increases with increasing $\mu$ for high temperatures.

\section{Superdiffusion $0<\mu<1$}

Figure 7 shows current $J$ as a function of temperature $T$ for superdiffusion $(0<\mu<1)$ at $\rho_{1}=\rho_{0}$. It is found that the curve is not smooth and there is a inflexion at which the negative current takes its maximum value. For low temperatures, the particles move to the left and the negative current increases with decreasing $\mu$. As temperature $T$ is increased, the current becomes to be positive and the positive current increases with decreasing $\mu$.

In order to explain the transport behavior for superdiffusion, the currents $j\left(F_{0}\right), j\left(-F_{0}\right)$ and $J$ versus temperature $T$ for $\mu=0.1$ are shown in Fig. 8. We can find from Eqs. (19) and (27) that there is a critical value of temperature

$$
T_{F_{0}}^{C}=-F_{0} \frac{\mu-1}{\mu}
$$

below which the local current $j\left(F_{0}\right)$ is zero. When $T$ is less than $T_{F_{0}}^{C}, j\left(F_{0}\right)$ is zero and $j\left(-F_{0}\right)$ is negative, so the current $J=\frac{1}{2} j\left(-F_{0}\right)$ is negative. When $T$ is more than $T_{F_{0}}^{C}, j\left(F_{0}\right)$ is more than $-j\left(-F_{0}\right)$, so the current $J$ is positive. As temperature $T$ is increased, $j\left(F_{0}\right)$ 


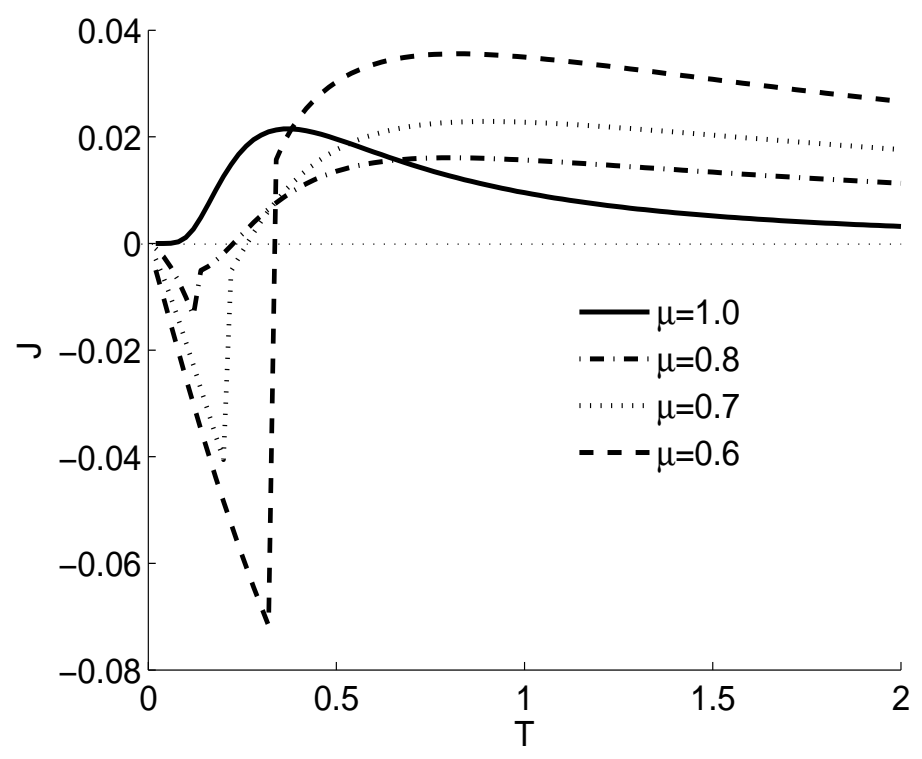

FIG. 7: Current $J$ as a function of temperature $T$ for different values of $\mu$ at $Q=1, \lambda=0.9$, $F_{0}=0.5, \rho_{0}=1.0$, and $\rho_{1}=1.0$.

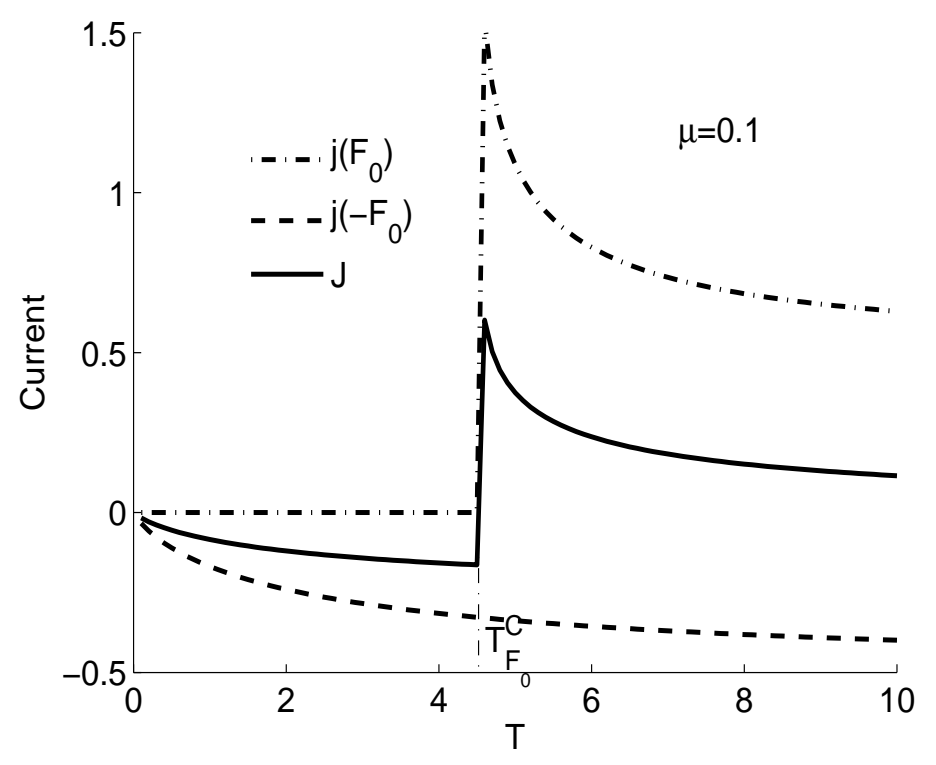

FIG. 8: Currents $j\left(F_{0}\right), j\left(-F_{0}\right)$ and $J$ versus temperature $T$ for $\mu=0.1$ at $Q=1, \lambda=0.9$, $F_{0}=0.5, \rho_{0}=1.0$, and $\rho_{1}=1.0$. The temperature at the inflexion is $T_{F_{0}}^{C}=4.5$. 
tends to $-j\left(-F_{0}\right)$, then the current $J$ goes to zero.

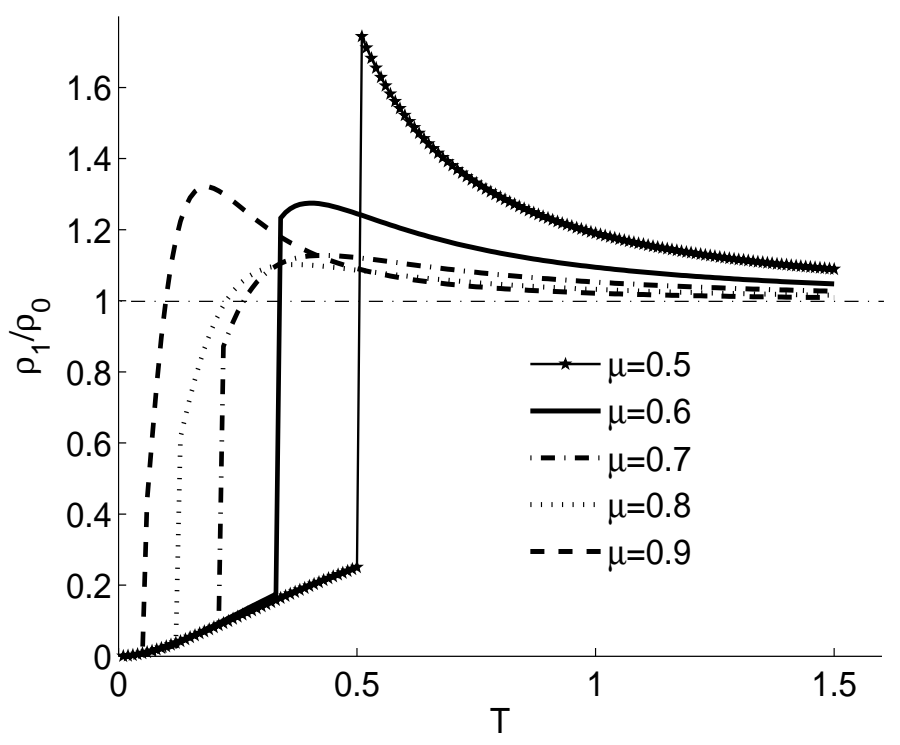

FIG. 9: Concentration ratio $\rho_{1} / \rho_{0}$ as a function of temperature $T$ for $J=0$ at $Q=1, \lambda=0.9$, $F_{0}=0.5, \rho_{0}=1.0$, and $\mu=0.5,0.6,0.7,0.8,0.9$.

Figure 9 shows the concentration ratio $\rho_{1} / \rho_{0}$ as a function of temperature $T$ for superdiffusion $(\mu=0.5,0.6,0.7,0.8$ and 0.9$)$. For low temperatures, $T$ is less than $T_{F_{0}}^{C}$ and $j\left(F_{0}\right)$ goes to zero, so the particles move to the left and $\rho_{1} / \rho_{0}<1$. As $\mu$ is decreased, the lowest temperature for pumping the particles to the right increases. Therefore, the pumping system in superdiffusive regime need a higher temperature than that in normal diffusive regime.

\section{CONCLUDING REMARKS}

In this study, we investigate a Brownian pump in nonlinear diffusive media with an unbiased external force. The pump is embedded in a finite region and bounded by two particle reservoirs. The analytical results are obtained in the adiabatic limit. In normal diffusive regime, current is a peaked function of temperature and concentration ratio for zero current decreases with increasing temperature. In subdiffusive regime, current is forbidden for low temperatures and negative for high temperatures. The concentration ratio for zero current decreases with increasing temperature, also. In superdiffusive regime, the transport compared with normal diffusion exhibits an opposite direction for low temperatures. There 
exists a finite temperature at which the concentration ratio takes its maximum value. For anomalous diffusion, there exist inflexions in the curves for $J$ vs $T$ and $\rho_{1} / \rho_{0}$. This is because a cut off of the stationary solution Eq. (18) restricts the attainable space.

\section{ACKNOWLEDGMENTS}

The work was supported by the National Natural Science Foundation of China under Grant No. 30600122 and GuangDong Provincial Natural Science Foundation under Grant No. 06025073.

[1] I. Kosztin and K. Schulten, Phys. Rev. Lett. 93, 238102 (2004).

[2] M. Buttiker, Z. Phys. B 68, 161 (1987).

[3] M. Moskalets and M. Buttiker, Phys. Rev. B 66, 035306 (2002).

[4] L. Arrachea, M. Moskalets, and L. Martin-Moreno, Phys. Rev. B 75, 245420 (2007).

[5] M. Rey, M. Strass, S. Kohler, P. Hanggi, and F. Sols, Phys. Rev. B 76, 085337 (2007).

[6] J. M. Sancho and A. Gomez-Marin, Europhysics Letters 86, 40002 (2009); J. M. Sancho and A. Gomez-Marin, Phys. Rev. E 77, 031108(2008).

[7] L. A. Peletier, in Application of Nonlinear Analysis in the Physical Sciences, edited by H. Ammam and N. Bazley (Pitman, Boston, 1981), p. 229.

[8] C. Anteneodo, Phys. Rev. E 76, 021102 (2007).

[9] E. K. Lenzi, C. Anteneodo, and L. Borland, Phys. Rev. E 63, 051109 (2001).

[10] J. L. Zhao, J. D. Bao, and W. P. Wei, J. Chem. Phys. 124, 024112 (2006).

[11] M. Muskat, The Flow of Homogeneous Fluids through Porous Media (McGraw-Hill, New York, 1937).

[12] M. E. Gurtin and R. C. MacCamy, Math. Biosci. 33, 35 (1977).

[13] Z. S. Khan and S. W. Morris, Phys. Rev. Lett. 94, 048002 (2005). 
[14] P. Rosenau, Phys. Rev. Lett. 74, 1056 (1995); A. Compte, D. Jou, and Y. Katayama, J. Phys. A 30, 1023 (1997).

[15] A. R. Plastino and A. Plastino, Physica A 222, 347 (1995); C. Tsallis and D. J. Bukman, Phys. Rev. E 54, R2197 (1996).

[16] P. Reimann, Phys. Rep. 361, 57 (2002).

[17] R. D. Astumian and P. Hanggi, Physics Today 55, 33(2002). 